\title{
A Randomized Phase I Study of Abemaciclib in Chinese Patients with Advanced and/or Metastatic Cancers
}

\author{
Jian Zhang ${ }^{1} \cdot$ Nong Yang $^{2}$ - Dongmei Ji ${ }^{1}$ Weina Shen ${ }^{1} \cdot$ Wenhua $\mathrm{Li}^{1} \cdot$ Rubing Han $^{3} \cdot$ Ning Wang $^{3} \cdot$ Haoxun Tao $^{3}$. \\ Sonya C. Chapman ${ }^{4}$. Amanda K. Sykes ${ }^{4}$. Wanli Zhang ${ }^{3}$. Xichun $\mathrm{Hu}^{1}$
}

Accepted: 29 December 2020 / Published online: 25 January 2021

(c) The Author(s) 2021

\begin{abstract}
Background Abemaciclib, a cyclin-dependent kinase 4 and 6 inhibitor, is approved in combination with endocrine therapy or as monotherapy for hormone receptor-positive and human epidermal growth factor receptor-2-negative (HR+/HER2-) advanced breast cancer outside of China.

Objective To evaluate the safety, tolerability, and pharmacokinetic (PK) profile of abemaciclib in Chinese patients with advanced and/or metastatic cancers.

Patients and methods A multicenter, open-label, phase I trial of abemaciclib in Chinese patients with advanced and/or metastatic cancers was conducted. Patients were randomized (1:1) to oral abemaciclib 150 or $200 \mathrm{mg}$ every $12 \mathrm{~h}$ on a 28 -day cycle. Safety analyses (primary outcome) included all patients receiving at least one dose of abemaciclib. PK and antitumor activity were also assessed.

Results Of the 26 patients randomized, 25 received abemaciclib $150 \mathrm{mg}(n=12)$ or $200 \mathrm{mg}(n=13)$. All 25 patients reported $\geq 1$ treatment-emergent adverse event (TEAE). The majority of TEAEs were Common Terminology Criteria for Adverse Events (CTCAE) Grade 1 or 2 in severity. The most frequent TEAEs of Grade $\geq 3$ were neutropenia (32\%) and thrombocytopenia (24\%). Four patients (16\%) discontinued treatment due to AEs. Abemaciclib exhibited slow absorption and clearance at single dose, with maximum concentrations achieved after around $6 \mathrm{~h}$ and an elimination half-life of approximately $24 \mathrm{~h}$. No complete response was observed, two patients (8\%) achieved partial response, with one confirmed responder, and the disease control rate was $68 \%(n=17)$.

Conclusions Abemaciclib was well tolerated and the safety and PK profiles in Chinese patients were comparable to those previously reported in non-Chinese populations. Preliminary antitumor activity was observed.
\end{abstract}

ClinicalTrials.gov identifier NCT02919696.

\section{Key Points}

This phase I study demonstrated an acceptable safety profile for abemaciclib in Chinese patients with advanced and/or metastatic cancer.

Jian Zhang and Nong Yang are co-first authors.

The safety profile and pharmacokinetic characteristics

Xichun $\mathrm{Hu}$

xchu2009@hotmail.com

1 Department of Medical Oncology, Fudan University Shanghai Cancer Center, Shanghai, China in Chinese patients were similar to those demonstrated previously in studies of non-Chinese populations.

Preliminary antitumor activity was observed, supporting further development of abemaciclib in Chinese patients.

2 Department of Medical Oncology, Hunan Cancer Hospital, Changsha, China

3 Eli Lilly and Company, Shanghai, China

4 Eli Lilly and Company, Windlesham, UK 


\section{Introduction}

During the cell cycle, the G1 restriction point controls entry into S-phase and is essential for maintaining control of cell division [1]. The cyclin-dependent kinases (CDK), CDK 4 and CDK 6 (hereafter designated CDK 4 and 6), participate in a complex with D-type cyclins to initiate the transition through the $\mathrm{G} 1$ restriction point. Over $90 \%$ of solid tumors have alterations in the CDK 4/6-cyclin D-INK4-retinoblastoma $(\mathrm{Rb})$ pathway through either increased CDK 4/6-cyclin D activity or mutations that attenuate the function of the INK4 or Rb proteins [2, $3]$. These alterations render cells less dependent on mitogenic signaling for proliferation, one of the hallmarks of cancer cells.

The CDK4/cyclin D1 complex is responsible for the phosphorylation and inhibition of the $\mathrm{Rb}$ tumor-suppressor gene product. Both in vitro and in vivo experiments in multiple cancer cell lines (colorectal, lung, glioblastoma, and blood cancers) have demonstrated that inhibition of phosphorylation of $\mathrm{Rb}$ protein results in $\mathrm{G} 1$ cell-cycle arrest [4].

Abemaciclib (LY2835219) is a potent and selective CDK 4 and 6 inhibitor, acting as an inhibitor of the ATPbinding domain of the kinases. It has 14 times greater potency against CDK 4 than against CDK 6 in vitro, and a higher selectivity for the cyclin D/CDK complex than other CDK 4 and 6 inhibitors [5]. Preclinical studies have suggested that short-term inhibition can result in reversible arrest and rebound induction of DNA synthesis, whereas continuous inhibition of CDK 4 and 6 leads to sustained cell-cycle arrest [6-8]. Abemaciclib can be dosed orally on a continuous schedule to achieve sustained target inhibition and demonstrates both durable cell-cycle inhibition and single-agent antitumor activity in xenograft models [9, 12]. Collectively, in patients with advanced solid tumors from multiple phase I and II studies, single-agent abemaciclib has demonstrated an acceptable safety profile and clinical activity against multiple human tumors, such as breast cancer and lung cancer [8, 10-12]. An efficacy benefit and acceptable safety profile has been shown for abemaciclib $150 \mathrm{mg}$ given every $12 \mathrm{~h}$ in women with hormone receptor-positive $(\mathrm{HR}+)$, human epidermal growth factor receptor-2-negative (HER2-) advanced breast cancer in combination with a non-steroidal aromatase inhibitor as first-line therapy, as well in combination with fulvestrant after progression on endocrine therapy as second-line therapy, based on the outcomes of the phase III MONARCH 3 study and MONARCH 2 study, respectively [13, 14].

During the dose-escalation part of the JPBA study, a phase I study of abemaciclib in mainly Caucasian patients with advanced cancer (NCT01394016), the maximum tolerated dose (MTD) of abemaciclib in patients with advanced solid tumors was established at $200 \mathrm{mg}$ given twice daily on a continuous schedule (every $12 \mathrm{~h}$ ) [12]. The overall safety profile and pharmacokinetic (PK) characteristics of the phase I study in Japanese patients (NCT02014129) were comparable to those observed in non-Japanese patients [15]. However, the safety and PK profile of abemaciclib has not been established in Chinese patients.

This phase I trial was designed to evaluate the safety, tolerability, and PK of abemaciclib in Chinese patients.

\section{Materials and Methods}

\subsection{Study Design}

This clinical study was a multicenter, open-label, phase I study of oral abemaciclib (LY2835219; Eli Lilly and Company, Indianapolis, IN, USA), in Chinese patients with advanced and/or metastatic cancers. The study was conducted at two clinical sites in China from 14 August 2017 (first patient enrolled) to data cut-off on 23 November 2018; 3 patients were still on treatment at the time of data cutoff. The last patient discontinued study treatment on 19 July 2019 by withdrawing consent from study participation. Written approval was provided by the Institutional Review Board, and the study was conducted in accordance with international ethics guidelines, including the Declaration of Helsinki, the International Conference on Harmonisation Good Clinical Practices Guideline [E6], and applicable laws and regulations. Informed consent was obtained from each patient before any protocol procedures or administration of study drug. This study was registered at ClinicalTrials.gov (NCT02919696).

\subsection{Study Population}

Eligible patients were $\geq 18$ years of age with histological or cytological evidence of cancer that was advanced and/or metastatic, and judged by the investigator to be an appropriate candidate for experimental therapy after available standard therapies had ceased to provide clinical benefit. Patients were required to have measurable disease defined by the Response Evaluation Criteria in Solid Tumors (RECIST version 1.1) [16]; an Eastern Cooperative Oncology Group (ECOG) performance status score $\leq 1$ [17]; adequate organ function; and to have discontinued all previous therapies (including chemotherapy, radiotherapy, immunotherapy, and investigational therapy) $\geq 21$ days for myelosuppressive agents or $\geq 14$ days for 
non-myelosuppressive agents prior to first dose of abemaciclib, and to have recovered from acute toxicities.

Patients were specifically excluded if they met any of the following exclusion criteria: medical history of presyncope or syncope of either unexplained or cardiovascular etiology, ventricular tachycardia, ventricular fibrillation, or sudden cardiac arrest, or had a baseline electrocardiogram (ECG) with any of the following abnormal findings: ventricular arrhythmia, evidence of acute myocardial ischemia, heart block (of any degree), or corrected QT interval (QTc) prolongation (defined as Bazett's corrected QT formula (QTcB) $\geq 450 \mathrm{~ms}$ ); presence of serious preexisting medical conditions that, in the judgment of the investigator, would preclude participation in this study; had active bacterial, fungal, and/or known viral infection; or had symptomatic central nervous system malignancy or metastasis.

\subsection{Randomization}

Patients were randomized 1:1 to receive oral abemaciclib at $150 \mathrm{mg}$ or $200 \mathrm{mg}$. Patients, physicians, and investigators were masked to treatment allocation.

\subsection{Treatment Plan}

Abemaciclib was administered at two dose levels (150 and $200 \mathrm{mg}$ ) based on previous escalation studies and safety evaluation data $[12,15,18,19]$. Abemaciclib was orally administered every $12 \mathrm{~h}$ on a 28-day cycle (Cycle 1: 32 days) at approximately the same time each day. In Cycle 1, the initial dose of abemaciclib was taken on day 1 to enable PK sampling over $72 \mathrm{~h}$ following a single dose; the remaining 55 doses were taken every $12 \mathrm{~h}$ on days 4-31. Enrollment was extended until at least 10 patients in each cohort had completed Cycle $1 \mathrm{PK}$ assessment in case of patient discontinuation.

Adherence to study drug was assessed at each visit by direct questioning, reviewing the patient diary, and counting returned capsules. Patients were required to take $\geq 75 \%$ of the intended dose in a cycle to be deemed adherent with study-drug administration. Treatment was discontinued in the following circumstances: disease progression, unacceptable toxicity, not recovering from toxicity within 14 days, patient death, non-compliance with treatment/procedures, or patient/investigator/physician/sponsor decision. Dose reductions and omissions were permitted both within a cycle and between cycles in accordance with pre-specified doseadjustment procedures. The start of a cycle was permitted to be delayed to allow sufficient time for recovery from toxicity possibly related to study drug.

\subsection{Treatment Assessments}

\subsubsection{Safety}

The primary objective of this study was to evaluate the safety and tolerability of abemaciclib in Chinese patients with advanced and/or metastatic cancers. In this study, safety analyses were conducted on all patients who had received at least one dose of the study drug. Adverse events (AEs) were graded using the Common Terminology Criteria for Adverse Events (CTCAE, version 4.0) for laboratory and non-laboratory parameters. Patient-reported adverse events were collected during the treatment period and laboratory results were collected per protocol-defined time points. Any clinically significant findings from laboratory, including chemistry, hematology, and urinalysis panels, as well as observation of vital signs, dose adjustments, and ECG, should also be reported to Lilly or its designee as an AE. Dose adjustments were allowed both within a cycle and between cycles by only one level $(50 \mathrm{mg})$ at a time.

\subsubsection{Pharmacokinetic Evaluation}

Plasma PK samples were collected at 1, 2, 4, 6, 8, 10, 24, 48, and $72 \mathrm{~h}$ after a single dose (Cycle 1, Day 1) and at 1, 2, 4, 6, 8,10 , and $24 \mathrm{~h}$ after multiple twice daily doses (Cycle 1, Day 31). Doses were held during these PK sampling periods to allow full characterization of the single dose and steady state PK profile. Pre-dose plasma PK samples were also collected on Days 1, 18, 25, and 31 of Cycle 1.

Plasma PK samples were analyzed for the concentrations of abemaciclib and its two major active metabolites, LSN2839567 [M2] and LSN3106726 [M20], using a validated liquid chromatography/tandem mass spectrometry assay.

PK parameter estimates for abemaciclib and its major equipotent metabolites, M2 and M20, were calculated by standard non-compartmental methods of analysis using WinNonlin ${ }^{\circledR}$ (Phoenix ${ }^{\circledR}$ version 8.1, Certara, NJ, USA). PK parameter estimates for total active analytes were computed by summating abemaciclib, M2, and M20.

\subsubsection{Efficacy}

Antitumor activity of abemaciclib, a secondary objective in this study, was analyzed by tumor response data, progression-free survival (PFS) data, and overall survival data. Tumor response and progression, including complete response (CR), partial response (PR), progressive disease (PD), stable disease (SD), and not evaluable, were evaluated using RECIST (version 1.1) [16]. Overall response rate (ORR) was defined as the percentage of enrolled patients with a best response of PR or CR. Disease control rate 
(DCR) was defined as the percentage of enrolled patients with a best response of PR, CR, or SD.

\subsubsection{Statistical Analysis}

More than 20 patients were enrolled in order to ensure 10 completers (patients who obtained the full set of PK samples for appropriate PK evaluation) in each dose cohort, without formal sample size calculation.

Details of patient disposition, demographics, and baseline disease characteristics were summarized for each cohort. Safety data (including vital signs and AEs) were summarized by cohort in frequency tables or by summary statistics. PK parameters were summarized using descriptive statistics by dose. Efficacy data were listed and summarized by cohort using frequency tables or summary statistics. All efficacy analyses were performed on the full analysis set of patients, which was defined as patients who received $\geq 1$ dose of study drug.

\section{Results}

\subsection{Patients}

A total of 26 patients were enrolled in the study: 12 in the $150 \mathrm{mg}$ cohort and 14 in the $200 \mathrm{mg}$ cohort. Among them, one patient randomized to the abemaciclib $200 \mathrm{mg}$ cohort was never treated. All treated patients had advanced and/or metastatic disease, with pathological diagnosis of breast cancer for 21 patients $(84.0 \%)$. Most patients had $\geq 3(48.0 \%)$ or $2(32.0 \%)$ organs involved. A majority of patients had been heavily treated previously with systemic therapy in the metastatic setting with a median of 4 regimens (range: $1-7$ ) (Table 1). Twenty-two patients (84.6\%) discontinued study treatment at data cut-off, and the most common reasons for treatment discontinuation were because of PD (61.5\%) and AEs (11.5\%).

\subsection{Safety}

For all treated patients $(n=25)$, the median number of cycles received per patient was 4.0 , the median duration of therapy was 18.6 weeks, and the median relative dose intensity was $83.3 \%$.

All 25 patients (100\%) reported at least one treatmentemergent AE (TEAE) of any grade and the safety profiles were comparable in both cohorts (Table 2). The most commonly reported TEAEs were diarrhea and neutropenia. Nineteen patients (76.0\%) reported TEAE of Grade $\geq 3$. Among them, 4 patients (16.0\%) had TEAE of Grade 4 in severity and 1 patient (4.0\%) died on treatment due to $\mathrm{AE}$ (respiratory failure) (Table 3).
Table 1 Patient demographics and disease characteristics

\begin{tabular}{|c|c|c|c|}
\hline Characteristic & $150 \mathrm{mg}(n=12)$ & $200 \mathrm{mg}(n=13)$ & Total $(n=25)$ \\
\hline \multicolumn{4}{|l|}{ Sex } \\
\hline Female & $11(91.7)$ & $11(84.6)$ & $22(88.0)$ \\
\hline Male & $1(8.3)$ & $2(15.4)$ & $3(12.0)$ \\
\hline Age, years & $55.6(11.5)$ & $52.0(8.8)$ & $53.7(10.1)$ \\
\hline \multicolumn{4}{|l|}{ Age category } \\
\hline$<65$ years & $8(66.7)$ & $13(100.0)$ & $21(84.0)$ \\
\hline$\geq 65$ years & $4(33.3)$ & 0 & $4(16.0)$ \\
\hline \multicolumn{4}{|l|}{ Primary tumor type } \\
\hline Breast & $10(83.3)$ & $11(84.6)$ & $21(84.0)$ \\
\hline Lung & $1(8.3)$ & $2(15.4)$ & $3(12.0)$ \\
\hline Other $^{\mathrm{a}}$ & $1(8.3)$ & 0 & $1(4.0)$ \\
\hline \multicolumn{4}{|c|}{ Numbers of organs involved at baseline } \\
\hline 1 & $3(25.0)$ & $2(15.4)$ & $5(20.0)$ \\
\hline 2 & $5(41.7)$ & $3(23.1)$ & $8(32.0)$ \\
\hline$\geq 3$ & $4(33.3)$ & $8(61.5)$ & $12(48.0)$ \\
\hline \multicolumn{4}{|c|}{ ECOG performance status } \\
\hline 1 & $12(100.0)$ & $13(100.0)$ & $25(100.0)$ \\
\hline $\begin{array}{l}\text { Prior anti-cancer } \\
\text { systemic } \\
\text { therapy }\end{array}$ & $12(100.0)$ & $13(100.0)$ & $25(100.0)$ \\
\hline \multicolumn{4}{|l|}{ Systemic therapy } \\
\hline \multicolumn{4}{|c|}{ Number of regimens for locally advanced/metastatic disease } \\
\hline 1 & $3(25.0)$ & $2(15.4)$ & $5(20.0)$ \\
\hline 2 & $1(8.3)$ & $2(15.4)$ & $3(12.0)$ \\
\hline 3 & $1(8.3)$ & $2(15.4)$ & $3(12.0)$ \\
\hline$\geq 4$ & $7(58.3)$ & $7(53.8)$ & $14(56.0)$ \\
\hline \multicolumn{4}{|c|}{ Type of systemic therapy for locally advanced/metastatic disease } \\
\hline $\begin{array}{l}\text { Chemo- } \\
\text { therapy }\end{array}$ & $12(100.0)$ & $13(100.0)$ & $25(100.0)$ \\
\hline $\begin{array}{c}\text { Endocrine } \\
\text { therapy }\end{array}$ & $9(75.0)$ & $8(61.5)$ & $17(68.0)$ \\
\hline $\begin{array}{l}\text { Target } \\
\text { therapy }\end{array}$ & $3(25.0)$ & $5(38.5)$ & $8(32.0)$ \\
\hline Other & $2(16.7)$ & $1(7.7)$ & $3(12.0)$ \\
\hline
\end{tabular}

Data are $n(\%)$ or mean (standard deviation), unless otherwise stated ECOG Eastern Cooperative Oncology Group

${ }^{a}$ Oral squamous-cell carcinoma

Diarrhea was the most common TEAE and reported in 20 patients $(80.0 \%)$, among whom 7 patients $(28.0 \%)$ reported diarrhea of Grade 2 and 4 patients reported diarrhea of Grade 3; diarrhea Grade 4 or 5 was not reported (Table 2 and 3). In terms of diarrhea incidences per patient, of the 20 patients experiencing diarrhea, 13 of the patients $(65.0 \%)$ had 1 or 2 episodes and 7 patients had 3 or more episodes. Supportive measures, including anti-diarrheal therapy and drinking fluid, were required to be initiated as early as possible. Diarrhea was well managed by antidiarrheal therapy and dose modification. Neutropenia was the most commonly reported TEAE of 
Table 2 Summary of treatment-emergent adverse events

\begin{tabular}{|c|c|c|c|}
\hline Preferred term & Total $(n=25)$ & $150 \mathrm{mg}$ every $12 \mathrm{~h}(n=12)$ & $\begin{array}{l}200 \mathrm{mg} \text { every } \\
12 \mathrm{~h}(n=13)\end{array}$ \\
\hline Patients with $\geq 1$ TEAE (any grade) & $25(100.0)$ & $12(100.0)$ & $13(100.0)$ \\
\hline Diarrhea & $20(80.0)$ & $9(75.0)$ & $11(84.6)$ \\
\hline Neutropenia $^{a}$ & $19(76.0)$ & $10(83.3)$ & $9(69.2)$ \\
\hline Leukopenia $^{\mathrm{b}}$ & $16(64.0)$ & $9(75.0)$ & $7(53.8)$ \\
\hline Decreased appetite & $15(60.0)$ & $9(75.0)$ & $6(46.2)$ \\
\hline Blood creatinine increased & $14(56.0)$ & $6(50.0)$ & $8(61.5)$ \\
\hline Malaise & $13(52.0)$ & $7(58.3)$ & $6(46.2)$ \\
\hline Anemia & $12(48.0)$ & $7(58.3)$ & $5(38.5)$ \\
\hline Thrombocytopenia $^{c}$ & $11(44.0)$ & $6(50.0)$ & $5(38.5)$ \\
\hline Nausea & $9(36.0)$ & $3(25.0)$ & $6(46.2)$ \\
\hline Cough & $8(32.0)$ & $4(33.3)$ & $4(30.8)$ \\
\hline Vomiting & $8(32.0)$ & $4(33.3)$ & $4(30.8)$ \\
\hline Alanine aminotransferase increased & $7(28.0)$ & $5(41.7)$ & $2(15.4)$ \\
\hline Bilirubin increased & $5(20.0)$ & $4(33.3)$ & $1(7.7)$ \\
\hline Localized edema & $5(20.0)$ & $3(25.0)$ & $2(15.4)$ \\
\hline
\end{tabular}

Data are $n(\%)$

The table shows TEAEs occurring in at least $25 \%$ of patients in either treatment group

CTCAE Common Terminology Criteria for Adverse Events, $h$ hours, TEAE treatment-emergent adverse events

${ }^{\text {a } C T C A E ~ t e r m ~ n e u t r o p h i l ~ c o u n t ~ d e c r e a s e d ~}$

${ }^{\mathrm{b}}$ CTCAE term white blood cell count decreased

${ }^{\mathrm{c}} \mathrm{CTCAE}$ term platelet count decreased

Table 3 Summary of treatment-emergent adverse events of Grade $\geq 3$

\begin{tabular}{|c|c|c|c|c|c|c|}
\hline \multirow[t]{2}{*}{ Preferred term } & \multicolumn{2}{|c|}{ Total $(n=25)$} & \multicolumn{2}{|c|}{$150 \mathrm{mg}(n=12)$} & \multicolumn{2}{|c|}{$200 \mathrm{mg}(n=13)$} \\
\hline & Grade 3 & Grade 4 & Grade 3 & Grade 4 & Grade 3 & Grade 4 \\
\hline Patients with $\geq 1$ TEAE of Grade $\geq 3^{\mathrm{a}}$ & $14(56.0)$ & $4(16.0)$ & $8(66.7)$ & $1(8.3)$ & $6(46.2)$ & $3(23.1)$ \\
\hline Neutropenia $^{\mathrm{b}}$ & $8(32.0)$ & 0 & $5(41.7)$ & 0 & $3(23.1)$ & 0 \\
\hline Thrombocytopenia $^{c}$ & $5(20.0)$ & $1(4.0)$ & $3(25.0)$ & $1(8.3)$ & $2(15.4)$ & 0 \\
\hline Diarrhea & $4(16.0)$ & 0 & $1(8.3)$ & 0 & $3(23.1)$ & 0 \\
\hline Leukopenia $^{\mathrm{d}}$ & $3(12.0)$ & 0 & 0 & 0 & $3(23.1)$ & 0 \\
\hline Anemia & $2(8.0)$ & 0 & $2(16.7)$ & 0 & 0 & 0 \\
\hline Hyponatraemia & $2(8.0)$ & 0 & $2(16.7)$ & 0 & 0 & 0 \\
\hline Embolism & $1^{\mathrm{e}}(4.0)$ & $3^{f}(12.0)$ & $1(8.3)$ & 0 & 0 & $3(23.1)$ \\
\hline Malaise & $1(4.0)$ & 0 & $1(8.3)$ & 0 & 0 & 0 \\
\hline Lung infection & $1(4.0)$ & 0 & $1(8.3)$ & 0 & 0 & 0 \\
\hline Blood bilirubin increased & $1(4.0)$ & 0 & $1(8.3)$ & 0 & 0 & 0 \\
\hline Muscular weakness & $1(4.0)$ & 0 & $1(8.3)$ & 0 & 0 & 0 \\
\hline Vomiting & 0 & $1(4.0)$ & 0 & $1(8.3)$ & 0 & 0 \\
\hline
\end{tabular}

Data are $n(\%)$

CTCAE Common Terminology Criteria for Adverse Events, TEAE treatment-emergent adverse events

${ }^{\mathrm{a} O n e}$ patient reported Grade $5 \mathrm{TEAE}$ of respiratory failure in $150 \mathrm{mg}$ cohort

${ }^{\mathrm{b}} \mathrm{CTCAE}$ term neutrophil count decreased

${ }^{\mathrm{c}} \mathrm{CTCAE}$ term platelet count decreased

${ }^{\mathrm{d}}$ CTCAE term white blood cell count decreased

${ }^{\mathrm{e}}$ One patient reported thromboembolic event

${ }^{\mathrm{f}}$ Two patients reported pulmonary embolism and one patient reported pulmonary artery embolism 
Grade $\geq 3$, occurring in 8 patients (32.0\%) (all with Grade 3 , Table 3). No febrile neutropenia was reported in the study. No patient discontinued study treatment due to diarrhea or neutropenia.

Four patients discontinued study treatment due to AEs: in the $150 \mathrm{mg}$ cohort, 1 patient reported leukopenia, and death due to respiratory failure was reported for the other patient while receiving treatment; in the $200 \mathrm{mg}$ cohort, 2 patients $(15.4 \%)$ reported pulmonary embolism.

In total, 6 patients $(24.0 \%)$ reported serious adverse events (SAEs). In the $150 \mathrm{mg}$ cohort, 1 patient reported anemia, thrombocytopenia, lung infection, and respiratory failure, and one patient reported alanine transaminase (ALT) increased. In the $200 \mathrm{mg}$ cohort, 3 patients reported pulmonary embolism and 1 patient reported urinary tract infection. All 3 patients in the $200 \mathrm{mg}$ cohort who reported SAEs of embolism had one or multiple risk factors, such as peripherally inserted central catheter (PICC) insertion and high body mass index (BMI), and received subsequent systemic anti-cancer treatment after appropriate anticoagulation treatment.

Six patients $(50.0 \%)$ from the $150 \mathrm{mg}$ cohort and 4 patients (30.8\%) from the $200 \mathrm{mg}$ cohort experienced dose reduction. The most frequent reason for dose reduction was neutropenia (in total).

No clinically meaningful findings were observed from ECG data or vital signs. Most of the laboratory abnormalities were of low grade and reversible.

\subsection{Pharmacokinetic Evaluation}

PK data for abemaciclib after single oral administration were available from 25 patients, of whom 21 had sufficient data to be included in the multiple dose PK analysis. PK samples were collected during Cycle 1 only, during which 5 patients in the $150 \mathrm{mg}$ cohort had a dose reduction from 150 to 100 $\mathrm{mg}$ abemaciclib and 2 patients in the $200 \mathrm{mg}$ cohort had a dose reduction from 200 to $150 \mathrm{mg}$ abemaciclib.

After a single dose of either 150 or $200 \mathrm{mg}$, abemaciclib exhibited slow absorption and clearance, reaching maximum concentrations $\left(C_{\max }\right)$ at $6-7 \mathrm{~h}$ post-dose $\left(t_{\max }\right)$, with an elimination half-life $\left(t_{1 / 2}\right)$ of approximately $24 \mathrm{~h}$ (Table 4 , Fig. 1a). PK exposures of abemaciclib and total active analytes increased in a dose-dependent manner, with extensive PK variability across all non-compartmental PK parameters.

After multiple doses of 150 or $200 \mathrm{mg}$ abemaciclib, absorption and elimination kinetics were comparable to single-dose PK, with similarly extensive variability (Table 4, Fig. 1b). Repeated dosing resulted in slightly increased exposures, with mean abemaciclib $C_{\max }$ values between 260 and $320 \mathrm{ng} / \mathrm{mL}$, compared to $200 \mathrm{ng} / \mathrm{mL}$ after a single dose. The area under the plasma concentration versus time curve during the dosing interval $\left(\mathrm{AUC}_{(0-12)}\right)$ increased by 1.57 - to 2.10-fold compared to single-dose values.

In addition to PK data for the parent drug, abemaciclib, the PK data for two major metabolites (M2 and M20) were also reported. Like abemaciclib, M2 and M20 exhibit comparable inhibitory potencies for CDK 4 and 6 . The PK of the sum of concentrations of abemaciclib, M2 and M20

Table 4 Summary of abemaciclib pharmacokinetic parameters (expressed as geometric mean unless otherwise stated) following a single oral dose on Day 1 and at steady state on Day 31 following twice-daily oral dosing of Cycle 1

\begin{tabular}{|c|c|c|c|c|c|}
\hline \multirow[t]{2}{*}{ Variable (geometric mean) } & \multicolumn{2}{|c|}{ Single dose (C1D1) } & \multicolumn{3}{|c|}{ Steady state (C1D31) } \\
\hline & & & $100 \mathrm{mg}$ every $12 \mathrm{~h}$ & $150 \mathrm{mg}$ every $12 \mathrm{~h}$ & $200 \mathrm{mg}$ every $12 \mathrm{~h}$ \\
\hline$N$ & 12 & 13 & 4 & 7 & 10 \\
\hline$C_{\max }[\mathrm{ng} / \mathrm{mL}(\% \mathrm{CV})]$ & $204(70)$ & $210(65)$ & $205(64)$ & $267(71)$ & $320(91)$ \\
\hline$t_{\max }[\mathrm{h}(\text { range })]^{\mathrm{a}}$ & $6.78(4.07-8.18)$ & $6.03(4.02-9.85)$ & $6.86(0.00-7.82)$ & $3.97(0.00-4.27)$ & $3.84(0.00-6.10)$ \\
\hline $\operatorname{AUC}_{(0-12)}[\mathrm{ng} \mathrm{h} / \mathrm{mL}(\% \mathrm{CV})]$ & $1700(75)$ & $1740(68)$ & $2190(65)$ & $2770(73)$ & $3270(101)$ \\
\hline $\operatorname{AUC}_{(0-\infty)}[\mathrm{ng} \mathrm{h} / \mathrm{mL}(\% \mathrm{CV})]$ & $6790(63)$ & $7580(74)$ & - & - & - \\
\hline $\mathrm{CL} / \mathrm{F}[\mathrm{L} / \mathrm{h}(\% \mathrm{CV})]$ & $22.1(63)$ & $26.4(74)$ & - & - & - \\
\hline$V_{\mathrm{ss}} / \mathrm{F}[\mathrm{L}(\% \mathrm{CV})]$ & $767(70)$ & $955(68)$ & - & - & - \\
\hline$t_{1 / 2}[\mathrm{~h}$ (range) $]$ & $22.4(15.2-34.8)$ & $22.8(15.6-33.2)$ & - & - & - \\
\hline$C_{\min , \mathrm{ss}}[\mathrm{ng} / \mathrm{mL}(\% \mathrm{CV})]$ & - & - & $159(66)$ & $202(72)$ & $238(103)$ \\
\hline $\mathrm{R}_{\mathrm{AUC}}$ & - & - & $\mathrm{NC}$ & $1.57(106)^{\mathrm{b}}$ & $2.10(69)$ \\
\hline
\end{tabular}

$A U C_{(0-\infty)}$ area under the plasma concentration-time curve from time zero to infinity, $A U C_{0-12}$ AUC during one 12-h dosing interval, $C$ cycle, $C L / F$ apparent systemic clearance, $C_{\max }$ maximum plasma concentration, $C_{\min }$ minimum plasma concentration, $C \mathrm{~V}$ coefficient of variation, $D$ day, $h$ hour(s), ss steady state, $t_{l / 2}$ terminal elimination half-life, $t_{\max }$ time of maximum plasma concentration, $V_{s s} / F$ apparent volume of distribution, $R_{A U C}$ accumulation ratio for $\mathrm{AUC}_{0-12}, N C$ not calculated

${ }^{\mathrm{a}}$ Median (range)

${ }^{\mathrm{b}} n=5$ 
Fig. 1 Abemaciclib mean plasma concentration-time following a single oral dose (a) and following twice-daily oral dosing (b). $h$ hours, $S D$ standard deviation
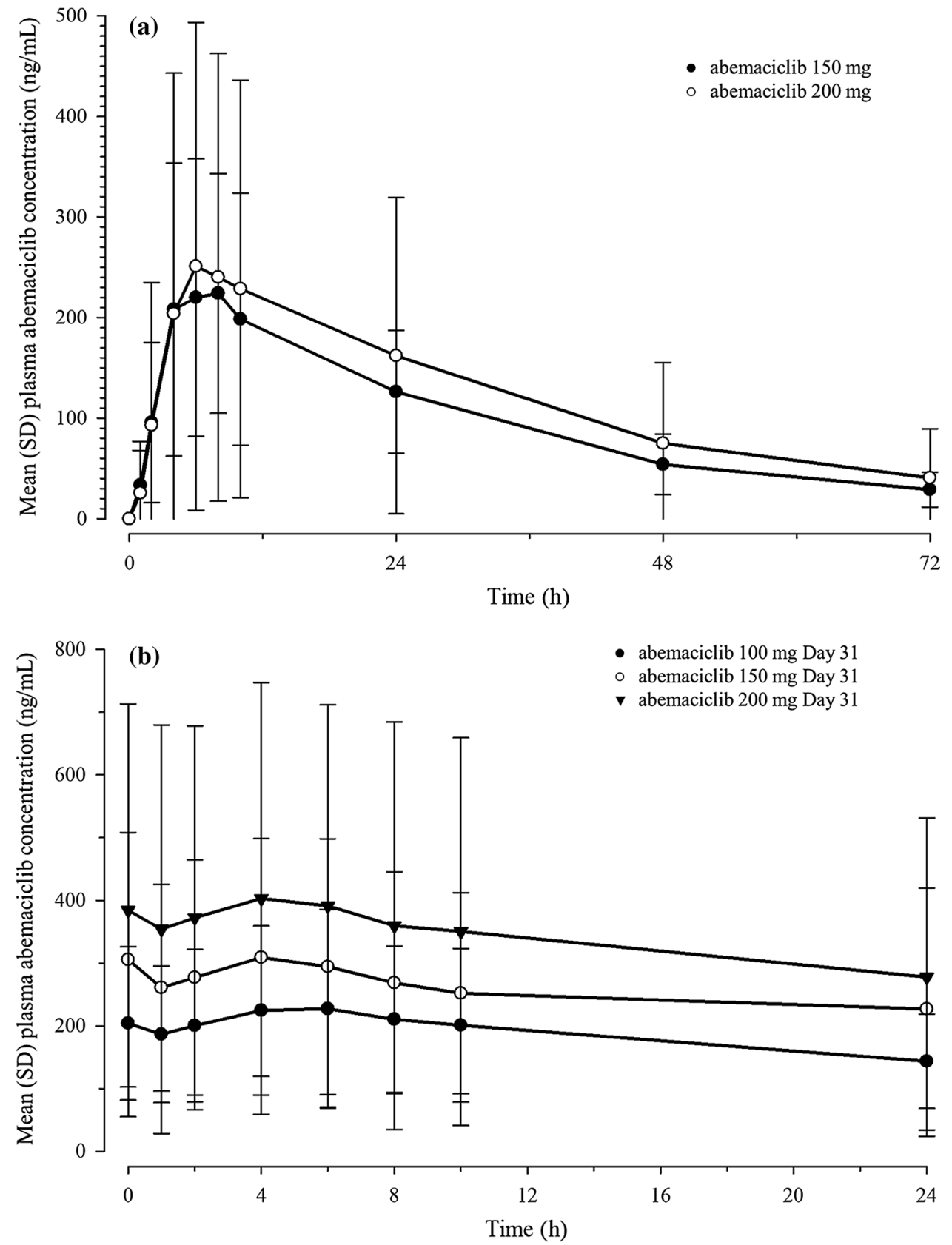

(hereafter termed "total active analytes") is reported in Table 5.

\subsection{Efficacy}

Of all treated patients, no CR was observed and 2 patients (8.0\%, 1 patient in each cohort) achieved PR, with 1 confirmed responder in the $150 \mathrm{mg}$ cohort (Table 6). The percentages of ORR and DCR were similar between the two cohorts. In total, 15 patients $(60.0 \%)$ had a best overall response (BOR) of SD and DCR was $68.0 \%(n=17)$. In this study, 21 enrolled patients $(84.0 \%)$ were pathologically diagnosed with breast cancer and evenly randomized to two cohorts, 14 patients $(66.7 \%)$ of whom had a BOR of SD and the DCR was $76.2 \%(n=16)$ (Table 6).

The median PFS was 4.31 months $(95 \%$ confidence interval [CI] 2.86-7.50) among the total treated population and 5.39 months (95\% CI 3.72-7.53) among the breast cancer patients. At the time of data cutoff, the OS data was not mature. 
Table 5 Summary of total active analyte pharmacokinetic parameters (expressed as geometric mean unless otherwise stated) following a single oral dose on Day 1 and at steady state on Day 31 following twice-daily oral dosing of Cycle 1

\begin{tabular}{|c|c|c|c|c|c|}
\hline \multirow[t]{2}{*}{ Variable (geometric mean) } & \multicolumn{2}{|c|}{ Single dose (C1D1) } & \multicolumn{3}{|l|}{ Steady state (C1D31) } \\
\hline & $150 \mathrm{mg}$ & $200 \mathrm{mg}$ & $100 \mathrm{mg}$ every $12 \mathrm{~h}$ & $150 \mathrm{mg}$ every $12 \mathrm{~h}$ & $200 \mathrm{mg}$ every $12 \mathrm{~h}$ \\
\hline$N$ & 12 & 13 & 4 & 7 & 10 \\
\hline$C_{\max }[\mathrm{nM}(\% \mathrm{CV})]$ & $620(59)$ & $624(59)$ & $905(57)$ & $1100(59)$ & $1360(100)$ \\
\hline$t_{\max }[\mathrm{h}(\text { range })]^{\mathrm{a}}$ & $6.8(4.07-8.18)$ & $6(4.02-10.47)$ & $6(4.12-7.82)$ & $4(0.00-4.27)$ & $3.9(0.00-7.53)$ \\
\hline $\operatorname{AUC}_{(0-12)}\left[\mathrm{nM}^{*} \mathrm{~h}(\% \mathrm{CV})\right]$ & $5350(63)$ & $5250(63)$ & $9770(58)$ & $11400(61)$ & $13800(107)$ \\
\hline $\operatorname{AUC}_{(0-\infty)}\left[\mathrm{nM}^{*} \mathrm{~h}(\% \mathrm{CV})\right]$ & $25400(55)$ & $27200(71)$ & - & - & - \\
\hline $\mathrm{CL} / \mathrm{F}[\mathrm{L} / \mathrm{h}(\% \mathrm{CV})]$ & $11.7(55)$ & $14.5(71)$ & - & - & - \\
\hline$V_{\mathrm{ss}} / \mathrm{F}[\mathrm{L}(\% \mathrm{CV})]$ & $488(61)$ & $615(65)$ & - & - & - \\
\hline$t_{1 / 2}[\mathrm{~h}$ (range) $]$ & $27.1(17.9-43)$ & $26.7(18.4-38.8)$ & - & - & - \\
\hline $\mathrm{C}_{\min , \mathrm{ss}}[\mathrm{nM}(\% \mathrm{CV})]$ & - & - & $740(60)$ & $843(61)$ & $1020(109)$ \\
\hline $\mathrm{R}_{\mathrm{AUC}}$ & - & - & $\mathrm{NC}$ & $2.12(106)^{b}$ & $2.91(67)$ \\
\hline
\end{tabular}

$A U C_{(0-\infty)}$ area under the plasma concentration-time curve from time zero to infinity, $A U C_{0-12}$ AUC during one 12 -h dosing interval, $C$ cycle, $C L / F$ apparent systemic clearance, $C_{\max }$ maximum plasma concentration, $C_{\min }$ minimum plasma concentration, $C \mathrm{~V}$ coefficient of variation, $D$ day, $h$ hour(s), ss steady state, $t_{1 / 2}$ terminal elimination half-life, $t_{\max }$ time of maximum plasma concentration, $V_{s s} / F$ apparent volume of distribution, $R_{A U C}$ accumulation ratio for $\mathrm{AUC}_{0-12}, N C$ not calculated

${ }^{a}$ Median (range)

${ }^{\mathrm{b}} n=5$

Table 6 Summary of best overall response

\begin{tabular}{|c|c|c|c|}
\hline Overall population & $150 \mathrm{mg}(n=12)$ & $200 \mathrm{mg}(n=13)$ & Total $(n=25)$ \\
\hline \multicolumn{4}{|l|}{ Best overall response $^{a}$} \\
\hline Complete response (CR) & 0 & 0 & 0 \\
\hline Partial response (PR) & $1(8.3)$ & $1(7.7)$ & $2(8.0)$ \\
\hline Stable disease (SD) & $7(58.3)$ & $8(61.5)$ & $15(60.0)$ \\
\hline Progressive disease (PD) & $2(16.7)$ & $2(15.4)$ & $4(16.0)$ \\
\hline Overall response rate $(\mathrm{CR}+\mathrm{PR})$ & $1(8.3)$ & $1(7.7)$ & $2(8.0)$ \\
\hline Disease control rate $(\mathrm{CR}+\mathrm{PR}+\mathrm{SD})$ & $8(66.7)$ & $9(69.2)$ & $17(68.0)$ \\
\hline Breast cancer treated population & $150 \mathrm{mg}(n 1=10)$ & $200 \mathrm{mg}(n 1=11)$ & Total $(n 1=21)$ \\
\hline \multicolumn{4}{|l|}{ Best overall response $^{a}$} \\
\hline Complete response (CR) & 0 & 0 & 0 \\
\hline Partial response (PR) & $1(10.0)$ & $1(9.1)$ & $2(9.5)$ \\
\hline Stable disease (SD) & $6(60.0)$ & $8(72.7)$ & $14(66.7)$ \\
\hline Progressive disease (PD) & $2(20.0)$ & $1(9.1)$ & $3(14.3)$ \\
\hline Overall response rate $(\mathrm{CR}+\mathrm{PR})$ & $1(10.0)$ & $1(9.1)$ & $2(9.5)$ \\
\hline Disease control rate $(\mathrm{CR}+\mathrm{PR}+\mathrm{SD})$ & $7(70.0)$ & $9(81.8)$ & $16(76.2)$ \\
\hline
\end{tabular}

Data are $n(\%) ; n 1$ means the number of breast cancer patients in population

RECIST response evaluation criteria in solid tumors

${ }^{\text {a }}$ Response criteria used was RECIST version 1.1

\section{Discussion}

In this phase I study conducted in Chinese patients with advanced and/or metastatic cancer, abemaciclib demonstrated an acceptable safety profile at both dose levels investigated. Diarrhea and hematological toxicities were manageable with supportive measures. The PK profile was characterized by slow absorption and high PK variability. Preliminary antitumor efficacy was observed for abemaciclib monotherapy in heavily pre-treated patients. 
The dose levels selected in this study were based on previous escalation studies and safety evaluation data. During the dose-escalation part of the JPBA study, the first in-human phase I study of abemaciclib, the MTD was established at $200 \mathrm{mg}$ given twice daily on a continuous schedule (every 12 h) [12]. In MONARCH 1, a phase II study of single agent abemaciclib, $200 \mathrm{mg}$ every $12 \mathrm{~h}$ was recommended for use in patients with HR+ and HER2metastatic breast cancer [8]. Safety data from the phase III studies MONARCH 2 and 3 demonstrated that the AE profile of $150 \mathrm{mg}$ abemaciclib administered every $12 \mathrm{~h}$ in combination with nonsteroidal aromatase inhibitors or fulvestrant was consistent with abemaciclib monotherapy $[13,14]$. Since the main objective of the present study was to evaluate the safety and tolerability of abemaciclib monotherapy in Chinese patients with advanced and/or metastatic cancers and to support further development of abemaciclib in Chinese patients, both doses of 150 and $200 \mathrm{mg}$ every $12 \mathrm{~h}$ were evaluated.

The overall safety profile of abemaciclib in Chinese patients demonstrated in this phase I study was similar to that observed in non-Chinese patients [8, 12, 15]. TEAEs were most commonly related to the gastrointestinal and hematopoietic systems, and most patients recovered with supportive measures. The safety profiles in both the $150 \mathrm{mg}$ and $200 \mathrm{mg}$ cohorts were comparable.

Of the 3 patients in the $200 \mathrm{mg}$ cohort who experienced an SAE of pulmonary embolism, all had 1 or several risk factors including high BMI, limited immobilization due to fracture, peripherally inserted central catheter insertion, and multiple metastases. Only 1 patient complained of slight chest distress, while the other 2 patients did not have any symptoms and the diagnoses were based on computerized tomography findings. All 3 patients received subsequent systemic treatment after recovering or having recovered by anticoagulation therapy. Considering the limited sample size, caution should be exercised in interpreting any incidence from the study. When evaluated in larger population, the incidence of embolism for abemaciclib in breast cancer was less than 5\%, including predominantly Chinese patients trial (MONARCH plus) [13, 14, 20]. Venous thromboembolic events have been reported with other CDK4 and 6 inhibitors [21]. Patients and physicians were suggested to monitor for signs and symptoms of venous thrombosis and pulmonary embolism and be managed as medically appropriate [22].

One patient died due to a respiratory failure AE. The patient suspended study drug treatment due to SAEs of Grade 3 anemia and thrombocytopenia during Cycle 1. Subsequently, the patient had multiple lacunar infarctions in the right cerebral area, lung infection, and developed new lung metastasis. The patient died due to type II respiratory failure caused by airway obstruction secondary to secretions.
The PK profiles of abemaciclib and total active analytes observed in Chinese patients included in the present study were consistent with the findings for abemaciclib monotherapy in the phase I JPBA study among a majority of Caucasian $(94.2 \%)$ cancer patients, and the phase II study MONARCH $1[12,23]$. The non-compartmental analysis demonstrated extensive variability in most PK parameters except the elimination half-life, which suggests underlying variability in absorption rather than clearance.

Antitumor activity of abemaciclib was evident in the Chinese population in this study, with 2 patients achieving PR (1 patient in each dose cohort). Notably, most patients enrolled in this study were heavily pre-treated with a median of 4 prior regimens of systemic therapy in the metastatic setting.

A possible limitation of this phase I study was, although the primary objective of the study was to evaluate the safety profile of abemaciclib in Chinese patients with advanced solid tumors, more than $80 \%$ of patients enrolled were breast cancer patients, which may impact the representativeness of all solid tumor types. This enrollment preference of the investigators may due to the fact that, at the time of study enrollment, abemaciclib was the only CDK4 and 6 inhibitor that demonstrated monotherapy activity in heavily pretreated breast cancer patients in the MONARCH 1 study and had received US Food and Drug Administration approval for this patient population.

\section{Conclusion}

This phase I study in Chinese patients with advanced and/ or metastatic cancer demonstrated that single-agent abemaciclib has an acceptable safety profile with evidence of antitumor activity. The safety profile and PK characteristics in Chinese patients were comparable to those observed in non-Chinese populations. These findings support ongoing development of abemaciclib in the Chinese population.

Acknowledgements We thank Dr. Shu Wang (Eli Lilly and Company) for her contribution to the preparation of the first draft and the final revision. Language editing under the direction of authors was provided by Dr. Jake Burrell $\left(\mathrm{CMPP}^{\mathrm{TM}}\right)$ from Rude Health Consulting Limited as funded by Eli Lilly, China. The authors are indebted to the patients who participated in this study, and their families.

\section{Declarations}

Funding This study was sponsored by Eli Lilly and Company, manufacturer/licensee of abemaciclib.

Conflict of interest Rubing Han, Ning Wang, Haoxun Tao, Sonya C. Chapman, Amanda K. Sykes, and Wanli Zhang are employees and minor shareholders of Eli Lilly and Company. The remaining authors have no conflicts of interest to declare. 
Ethics approval Written approval was provided by the Institutional Review Board, and the study was conducted in accordance with international ethics guidelines, including the Declaration of Helsinki, the International Conference on Harmonisation Good Clinical Practices Guideline [E6], and applicable laws and regulations.

Consent to participate Informed consent was obtained from each patient before any protocol procedures or administration of study drug.

\section{Consent for publication N/A.}

Availability of data and material Lilly provides access to all individual participant data collected during the trial, after anonymization, with the exception of pharmacokinetic or genetic data. Data are available to request 6 months after the indication studied has been approved in the US and EU and after primary publication acceptance, whichever is later. No expiration date of data requests is currently set once data are made available. Access is provided after a proposal has been approved by an independent review committee identified for this purpose and after receipt of a signed data sharing agreement. Data access should comply with local laws and regulations. Data and documents, including the study protocol, statistical analysis plan, clinical study report, blank or annotated case report forms, will be provided in a secure data sharing environment. For details on submitting a request, see the instructions provided at www.vivli.org.

\section{Code availability N/A.}

Author contributions The funder of the study (Eli Lilly and Company) had a role in study design, data collection, data analysis, data interpretation (in collaboration with all authors), and writing of the report. The corresponding author had full access to all the data in the study and takes responsibility for the integrity of the data and the accuracy of the data analysis.

Open Access This article is licensed under a Creative Commons Attribution-NonCommercial 4.0 International License, which permits any non-commercial use, sharing, adaptation, distribution and reproduction in any medium or format, as long as you give appropriate credit to the original author(s) and the source, provide a link to the Creative Commons licence, and indicate if changes were made. The images or other third party material in this article are included in the article's Creative Commons licence, unless indicated otherwise in a credit line to the material. If material is not included in the article's Creative Commons licence and your intended use is not permitted by statutory regulation or exceeds the permitted use, you will need to obtain permission directly from the copyright holder. To view a copy of this licence, visit http://creativecommons.org/licenses/by-nc/4.0/.

\section{References}

1. Ortega S, Malumbres M, Barbacid M. Cyclin D-dependent kinases, INK4 inhibitors and cancer. Biochim Biophys Acta. 2002;1602(1):73-87. https://doi.org/10.1016/s0304 $-419 x(02) 00037-9$.

2. Malumbres M, Barbacid M. To cycle or not to cycle: a critical decision in cancer. Nat Rev Cancer. 2001;1(3):222-31. https:// doi.org/10.1038/35106065.

3. Altenburg JD, Farag SS. The potential role of PD0332991 (Palbociclib) in the treatment of multiple myeloma. Expert Opin Investig Drugs. 2015;24(2):261-71. https://doi.org/10.1517/13543 784.2015.993753.
4. Corona SP, Generali D. Abemaciclib: a CDK4/6 inhibitor for the treatment of HR+/HER2- advanced breast cancer. Drug Des Devel Ther. 2018;12:321-30. https://doi.org/10.2147/DDDT. S137783.

5. Lallena MJ, Boehnke K, Torres R, et al. Abstract 3101: In-vitro characterization of Abemaciclib pharmacology in ER+ breast cancer cell lines. Cancer Res. 2015;75(15_suppl):3101. https:// doi.org/10.1158/1538-7445.AM2015-3101.

6. Gelbert LM, Cai S, Lin X, Sanchez-Martinez C, Del Prado M, Lallena MJ, et al. Preclinical characterization of the CDK4/6 inhibitor LY2835219: in-vivo cell cycle-dependent/independent anti-tumor activities alone/in combination with gemcitabine. Invest New Drugs. 2014;32(5):825-37. https://doi.org/10.1007/ s10637-014-0120-7.

7. Torres-Guzman R, Calsina B, Hermoso A, Baquero C, Alvarez B, Amat J, et al. Preclinical characterization of abemaciclib in hormone receptor positive breast cancer. Oncotarget. 2017;8(41):69493-507. https://doi.org/10.18632/oncotarget .17778 .

8. Dickler MN, Tolaney SM, Rugo HS, Cortes J, Dieras V, Patt D, et al. MONARCH 1, a phase II study of Abemaciclib, a CDK4 and CDK6 inhibitor, as a Single Agent, in Patients with Refractory HR(+)/HER2(-) Metastatic Breast Cancer. Clin Cancer Res. 2017;23(17):5218-24. https://doi.org/10.1158/1078-0432. CCR-17-0754.

9. Gelbert LM, Cai S, Lin X, Sanchez-Martinez C, del Prado M, Lallena MJ, et al. Identification and characterization of LY2835219: A potent oral inhibitor of the cyclindependent kinases 4 and 6 (CDK4/6) with broad in vivo antitumor activity. Mol Cancer Ther. 2011;10(11_suppl):B233. https://doi.org/10.1158/1535-7163. TARG-11-B233.

10. Patnaik A, Rosen LS, Tolaney SM, Tolcher AW, Goldman JW, Gandhi L, et al. LY2835219, a novel cell cycle inhibitor selective for CDK4/6, in combination with fulvestrant for patients with hormone receptor positive $(\mathrm{HR}+)$ metastatic breast cancer. J Clin Oncol. 2014;32(15_suppl):534. https://doi.org/10.1200/ jco.2014.32.15_suppl.534.

11. Tolaney SM, Rosen LS, Beeram M, Goldman JW, Gandhi L, Tolcher AW, et al. Abstract P5-19-13: Clinical activity of abemaciclib, an oral cell cycle inhibitor, in metastatic breast cancer. Cancer Res. 2015;75(9_suppl):P5-19-13. https://doi. org/10.1158/1538-7445.SABCS14-P5-19-13.

12. Patnaik A, Rosen LS, Tolaney SM, Tolcher AW, Goldman JW, Gandhi L, et al. Efficacy and safety of abemaciclib, an inhibitor of CDK4 and CDK6, for patients with breast cancer, nonsmall cell lung cancer, and other solid tumors. Cancer Discov. 2016;6(7):740-53. https://doi.org/10.1158/2159-8290. CD-16-0095.

13. Goetz M, Toi M, Campone M, Paluch-Shimon S, et al. MONARCH 3: abemaciclib as initial therapy for advanced breast cancer. J Clin Oncol. 2017;35(32):3638-46. https://doi.org/10.1200/ JCO.2017.75.6155.

14. Sledge GW Jr, Toi M, Neven P, Sohn J, Inoue K, Pivot X, et al. MONARCH 2: abemaciclib in combination with fulvestrant in women with HR+/HER2- advanced breast cancer who had progressed while receiving endocrine therapy. J Clin Oncol. 2017;35(25):2875-84. https://doi.org/10.1200/JCO.2017.73.7585.

15. Fujiwara $Y$, Tamura K, Kondo S, Tanabe Y, Iwasa S, Shimomura A, et al. Phase 1 study of abemaciclib, an inhibitor of CDK 4 and 6 , as a single agent for Japanese patients with advanced cancer. Cancer Chemother Pharmacol. 2016;78(2):281-8. https://doi. org/10.1007/s00280-016-3085-8.

16. Eisenhauer EA, Therasse P, Bogaerts J, Schwartz LH, Sargent D, Ford R, et al. New response evaluation criteria in solid tumours: revised RECIST guideline (version 1.1). Eur J Cancer. 2009;45(2):228-47. https://doi.org/10.1016/j.ejca.2008.10.026. 
17. Oken MM, Creech RH, Tormey DC, Horton J, Davis TE, McFadden ET, et al. Toxicity and response criteria of the Eastern Cooperative Oncology Group. Am J Clin Oncol. 1982;5(6):649-55.

18. Tolaney SM, Beeram M, Beck JT, Conlin AK, Dees EC, Dickler MN, Helsten TL, et al. A phase Ib study of abemaciclib with therapies for metastatic breast cancer. J Clin Oncol. 2015;33(15_ suppl):522. https://doi.org/10.1200/jco.2015.33.15_suppl.522.

19. Goetz M, Beeram M, Beck T, Conlin A, Dees E, Dickler M, et al. Abstract P4-13-25: Abemaciclib, an inhibitor of CDK4 and CDK6, combined with endocrine and HER2-targeted therapies for women with metastatic breast cancer. J Cancer Res. 2016;76(4 suppl):P4-13-25. https://doi.org/10.1158/1538-7445.SABCS 15-P4-13-25.

20. Zhang QY, Sun T, Yin YM, et al. MONARCH plus: abemaciclib plus endocrine therapy in women with HR+/HER2- advanced breast cancer: the multinational randomized phase III study. Ther Adv Med Oncol. 2020;12:1-14. https://doi.org/10.1177/17588 35920963925.

21. Gervaso L, Montero AJ, Jia X, Khorana AA. Venous thromboembolism in breast cancer patients receiving cyclin-dependent kinase inhibitors. J Thromb Haemost. 2020;18:162-8. https://doi. org/10.1111/jth. 14630 .

22. https://www.accessdata.fda.gov/drugsatfda_docs/label /2018/208855s000lbl.pdf.

23. O'Shaughnessy J, Chigutsa E, Kambhampati SRP, Sykes A, Frenzel M, Nanda S, Koustenis A, Smith I, Turner PK. Abemaciclib exposure-response relationship in patients with metastatic breast cancer in MONARCH 1. Cancer Res. 2017;77(4_suppl):P6-11-11. https://doi.org/10.1158/1538-7445.SABCS16-P6-11-11. 